\section{Wilderness Airway Management: Keeping An Eye on the Ball}

\section{To the Editor:}

The recent report on wilderness airways was an interesting case, and the patient's life was saved by skilled and attentive care. ${ }^{1}$

However, I disagree with the authors' conclusion that this suggests a need for more widespread training in wilderness operative airways. The most important element of this case was the sound reasoning behind deciding on an operative airway, and this core aspect deserves emphasis. In most circumstances, airways can be safely managed with less invasive means, thus limiting additional risk while proceeding with physiologic stabilization and evacuation. Too often, people are blinded by the opportunity to apply a procedural skill they have learned instead of concentrating on critical decision-making. Many more lives are rescued with proper use of bag-mask and suction than with intubation or tracheotomy. This general principle has recently been supported in the literature. ${ }^{2}$

On a separate note, the report omits any description of what happened with the airway following hospital admission. When surgeons evaluated the airway in a controlled environment, what did they find? In particular, was the tube confirmed to traverse the cricothyroid membrane, or had it taken an unintended course? Was his voice affected? Was the cricothyrotomy converted to a formal tracheostomy, or was he immediately decannulated? If the technical success of the procedure is to be lauded, these details must be known. A wilderness provider may argue that these elements are beyond their scope, but without follow-up on our interventions, none of us can truly know how accurate or successful our actions are.

Ramin Jamshidi, MD

Pediatric General \& Thoracic Surgery Phoenix Children's Hospital

Phoenix, AZ

\section{References}

1. Wharton DR, Bennett BL. Surgical Cricothyrotomy in the Wilderness: A Case Report. Wilderness Environ Med. 2013; 24:12-14.

2. Hasegawa K, Hiraide A, Chang Y, Brown DFM. Association of prehospital advanced airway management with neurologic outcome and survival in patients with out-ofhospital cardiac arrest. JAMA. 2013;309:257.

\section{In Reply to Dr Jamshidi's Letter}

\section{To the Editor:}

We would like to address the 2 general observations made by Dr Jamshidi about widespread training of the invasive skill of cricothyrotomy, and the airway clinical outcome after admission to the hospital. Our concern for enhanced cricothyrotomy training is based on recent data that identify the magnitude of the cricothyrotomy failure rates in the prehospital setting, which is primarily on the battlefield but with direct application to the wilderness setting. ${ }^{1}$ Medical care in any austere environment can be challenging and is amplified by limitations in medical resources, manpower, and delayed evacuation to definitive care. Recently, 2 articles point out the limitations in cricothyrotomy training and have made recommendations for enhancing how this is conducted in effort to decrease the cricothyrotomy failure rate. ${ }^{2,3}$

Cricothyrotomy is the last step in the difficult airway algorithm, and it is now being performed less often in the prehospital setting and the emergency department because there are bridging devices, for example, bagvalve mask and supraglottic airways, that are successful in supporting ventilation after endotracheal intubation failed attempts. Unfortunately, in our case report, an attempt to provide ventilation with a bag-valve mask was futile due to the lack of an effective facial seal. With this level of facial trauma, the use of a bag-valve mask is reported to worsen hypoxemia by pushing blood, debris, and tissue into the lower airways, and potentially compounds the damage to unstable facial structures. ${ }^{4}$

Further, the need for a definitive airway in the austere setting ultimately comes from a different etiology than that seen in the normal civilian setting. Whereas primary cardiac arrest in adults and primary respiratory arrest in children form the bulk of airway problems in this setting, in the wilderness it can be expected that airway difficulty would primarily be secondary to trauma or to anaphylaxis. Head, maxillofacial, and neck trauma can make a surgical airway the primary life-saving maneuver while significant airway edema may make any oral or nasal approach to the airway overly difficult if not impossible without the benefit of rapid sequence intubation.

With regard to the hospital admission airway outcome, the patient was changed over to tracheal intubation at the trauma center emergency department shortly after arrival. He received facial surgery and the first of several orthopedic operations within the first few hours. Once his facial fractures were stabilized, he was extubated after surgery. Within days, the rescue team physician (D.W.) visited him on the general patient floor. For- 